\title{
Nanoemulsions Target to Ectopic Lymphoids in Inflamed Joints to Restore Immune Tolerance in Rheumatoid Arthritis
}

Chenglong Li, Xiaoyan Chen, Xianjin Luo, Hairui Wang, Yining Zhu, Guangshen Du, Wenfei Chen, Zhengjun Chen, Xinyan Hao, Zhirong Zhang, and Xun Sun*

Key Laboratory of Drug Targeting and Drug Delivery Systems, Ministry of Education, West China School of Pharmacy, Sichuan University, Chengdu 610041, P.R. China.

\section{Corresponding Authors}

Email: sunxun@scu.edu.cn.

\section{Materials and Methods}

Reagents: Egg yolk lecithin (E80) was purchased from Lipoid (Germany). Soybean oil (SO) and medium chain triglyceride oil (MCT) were purchased from AVIC $\begin{array}{llll}\text { Pharmaceutical } & \text { (Tieling, } & \text { China). } & \text { CitP }\end{array}$ (VCitLCitSSVESTCitGRSCitPAPPPACitGLT-K) was synthesized by GL Biochem (Shanghai, China). Rapamycin was obtained from Meilun Biotechnology (Dalian, China). The cell counting kit-8 was purchased from Abmole Bioscience (Shanghai, China). All ELISA kits for cytokines were purchased from eBioscience (USA). Monoclonal antibodies conjugated with fluorescence were purchased from eBioscience or Biolegend (USA). DSPE-PEG2000 was purchased from AVT (Shanghai, China). 
Preparation and Characterization of NEs@CitP/Rapa: NEs@CitP/Rapa were fabricated by using homogenizing method with modifications. Briefly, $0.25 \mathrm{~g} \mathrm{SO}, 0.25$ $\mathrm{g}$ MCT and $0.5 \mathrm{~g}$ egg yolk lecithin were mixed uniformly, after which water phase was added to form initial emulsion. Next, the initial emulsion was homogenized with a highpressure homogenizer (AH-NANO, ATS, Canada) at 1000 bar for 10 min to produce injectable lipid emulsions (ILEs). $2 \mathrm{~mL}$ of ILEs were well mixed with $10 \mathrm{mg}$ of DSPEPEG2000. Then $30 \mu \mathrm{L}(200 \mathrm{mM})$ of ferric chloride aqueous solution was added to the $0.1 \mathrm{~mL}$ above PEGylated ILEs, and slowly dropped into the sodium hydroxide solution $(0.1 \mathrm{M})$ to adjust the $\mathrm{pH}$ to neutral, and the blank NEs were obtained. In order to form drug-loaded nano-emulsions, rapamycin was added to appropriate volume of blank vehicle and stirred continuously until the rapamycin was encapsulated in oil phase, and then the peptide aqueous solution was added into NEs@Rapa with well-mixed and incubation at $37{ }^{\circ} \mathrm{C}$ for half an hour to encapsulate peptide. The NEs@Did were prepared according to the preparation process of NEs@Rapa.

To quantify encapsulation efficiency of rapamycin, drug loaded particles were separated from free drug by Sephadex G-75, and NEs@Rapa solution was demulsificated in mixed solvents of $1 \%$ Triton/THF/ethanol $(1: 2.5: 2.5, \mathrm{v} / \mathrm{v} / \mathrm{v})$ and sonicated for $10 \mathrm{~min}$ to extract rapamycin completely. Then HPLC was used to determine the rapamycin concentration, and measurement was performed at $35^{\circ} \mathrm{C}$ on a $\mathrm{C}_{18}$ column $(5 \mu \mathrm{m}, 250 \times 4.6 \mathrm{~mm}$, Kromasil, Sweden $)$. The mobile phase was acetonitrile/water $(90: 10, \mathrm{v} / \mathrm{v})$, and wavelength was $278 \mathrm{~nm}$. To detect encapsulation efficiency of CitP peptide, free peptide in NEs@CitP solution was directly determined 
by gel filtration chromatography. The chromatographic column was TSKgel G2000SWXL $(300 \times 7.8 \mathrm{~mm}, 5 \mu \mathrm{m}$, TSK, Japan), and the mobile phase and wavelength were deionized water and $220 \mathrm{~nm}$, respectively.

Hydrodynamic diameter and zeta potential of NEs@CitP/Rapa were determined at $25^{\circ} \mathrm{C}$ by dynamic light scattering on a Malvern Zetasizer Nano-ZS (Malevern Zeta Sizer Nano ZS90, UK). Particle size and morphology were further characterized via transmission electron microscopy (H-600, Hitachi, Japan) after being stained by 2\% (w/v) phospho-tungstic acid aqueous solution.

Cellular- and Intracellular-stained for Flow Cytometry: Cell samples were washed by $1 \times$ PBS for once, then cell surface molecules were stained with monoclonal antibody labeled by fluorescence for $40 \mathrm{~min}$ at room temperature according to the manufacturer's instructions. Then cells were washed twice and resuspended in PBS by flow cytometry (FACS Celeata, BD Biosciences, USA). For intracellular-stained, cells need to be treated with fixation reagent and permeabilization reagent according to instructions after treated with the antibody against surface molecules (Intracellular Fixation \& Permeabilization Buffer Set, eBioscience, USA), and then the cells were stained with monoclonal antibodies, washed twice, and resuspended in PBS for analysis. The cells were intracellular-stained by Foxp3 using special kit (Foxp3/Transcription Factor Staining Buffer Set, eBioscience, USA). All flow cytometry data was analyzed on the FlowJo 10 (BD bioscience, USA).

Cell Uptake and BMDC Activation: Method to acquire bone marrow-derived DCs (BMDCs) has been previously described. ${ }^{1,2}$ BMDCs were exposed to medium 
containing particles encapsulating FITC-labeled CitP, soluble FITC-labeled CitP, or equal volume of sterile PBS for $1 \mathrm{~h}$. After incubation, cells were collected, rinsed with PBS and stained. Finally, cells were resuspended and measured by a flow cytometer. Immature BMDCs were collected and plated in a 24 wells $\left(1 \times 10^{6}\right)$ plate for $3 \mathrm{~h}$ with stimulation of LPS ( $4 \mu \mathrm{g} /$ well), and then the cells were incubated with PBS, Free CitP, NEs@CitP/Rapa, Free Rapa, Free Fe, or NEs@Rapa at the same dosage $(($ Rapamycin $=1 \mu \mathrm{g} / \mathrm{well}, \mathrm{CitP}=2 \mu \mathrm{g} / \mathrm{well}))$ for another $20 \mathrm{~h}$. Finally, cells were collected, washed by PBS, stained with fluorescence-labelled antibodies against CD40, CD86, and MHCII according to the mentioned protocols, and then analyzed by flow cytometry.

In Vitro Cytotoxic Assay: RAW264.7 cells were used to study the cytotoxicity of iron with various concentrations in NEs. Briefly, cells were cultured in RPMI-1640 medium and supplemented with $10 \%$ fetal bovine serum, $100 \mathrm{units} / \mathrm{ml}$ penicillin, and $100 \mathrm{mg} / \mathrm{ml}$ streptomycin at $37{ }^{\circ} \mathrm{C}$ in a humidified atmosphere containing $5 \% \mathrm{CO}_{2}$. NEs solution was diluted by medium (with ferric ion concentrations of $0.3,1.4,2.8,5.6$, and 8.3 $\mu \mathrm{g} / \mathrm{mL}$ ) and then added to each well. After $24 \mathrm{~h}, \mathrm{CCK}-8$ reagent was added to each well. The absorbance was measured using a microtiter-plate spectrophotometer at $450 \mathrm{~nm}$ after $2 \mathrm{~h}$ incubation. Cell viability was calculated by the following equation: cell viability $(\%)=(\mathrm{A}$ treatment- $\mathrm{A}$ blank control $) /(\mathrm{A}$ without treatment $-\mathrm{A}$ blank control $) \times 100$.

DC2.4 cells were used to study the cytotoxicity of CitP peptide with various concentrations. CitP peptide was diluted by medium $(0,65,130$, and $200 \mu \mathrm{M})$ and then added to each well. After 48 h, CCK-8 reagent was added to each well. The absorbance 
was measured after $2 \mathrm{~h}$ incubation. DC2.4 cells were also used to study the cytotoxicity of rapamycin in free form and NEs@Rapa at various concentrations.

Animal Models: BALB/c mice aged $6-8$ weeks were purchased from Dashuo Biotechnology Company (Chengdu, China). DBA/1 mice aged 6 - 8 weeks were purchased from Beijing HFK Bioscience Company (Beijing, China). All animals were kept in $12 \mathrm{~h}$ light/12 $\mathrm{h}$ dark periods under pathogen-free conditions with continuous access to food and water, and all animal experiments were performed following guidelines approved by the ethics committee of Sichuan University.

For Adjuvant-Induced Arthritis (AIA) mice model, male BALB/c animals were subcutaneously injected $0.1 \mathrm{~mL}$ of complete Freund's adjuvant (CFA) containing $1 \mathrm{mg} / \mathrm{mL}$ of heatkilled M. tuberculosis (7008, Chondrex, USA) into the footpad of a hind-paw. Severe and acute inflammation was observed within 30 min after injection, peaks within 14 to 16 days, and often persists for 30 days.

For Collagen-Induced Arthritis (CIA) mice model, male DBA/1 $\left(\mathrm{H}-2^{\mathrm{q}}\right)$ animals were subcutaneously injected $0.1 \mathrm{~mL}$ (100 mg collagen/mouse) of emulsion containing equal volume of bovine type-II collagen solution ( $2 \mathrm{mg} / \mathrm{mL}, 20022$, Chondrex, USA) and complete Freund's adjuvant ( $4 \mathrm{mg} / \mathrm{mL}, 7001$, Chondrex, USA) at the base of the tail. After 21 days of the first immunization, the boost immunization was injected 0.1 $\mathrm{mL}$ of emulsion containing equal volume of bovine type-II collagen solution $(2 \mathrm{mg} / \mathrm{mL})$ and incomplete Freund's adjuvant in the same manner. Arthritis developed 28 - 35 days after the first immunization.

In Vivo and Ex Vivo Fluorescence Imaging: The AIA mice model was constructed 
according to the above protocol. To study the consequence of biodistribution caused by various contents of PEG. NEs with various DSPE-mPEG 2000 contents $(0,0.5 \%, 5.0 \%)$ encapsulated with Did ( $1 \mu \mathrm{g} /$ mouse) were injected intravenously into AIA mice on day 3 after AIA-induction. The paws and major organs of the mice were harvested at $53 \mathrm{~h}$ post-injection for ex vivo imaging.

To study the biodistribution of NEs, the NEs encapsulated with Did ( $1 \mu \mathrm{g} / \mathrm{mouse})$ was injected intravenously into AIA mice on day 3 after AIA-induction. The control group received the same dosage of free Did dissolved in 1,2-propanediol aqueous solution $(5: 100, \mathrm{v} / \mathrm{v})$. The biodistribution were acquired by an IVIS imaging system at different time points. The major organs and inflamed paws were collected at $52 \mathrm{~h}$ postinjection for ex vivo imaging. The ROI fluorescence analysis was performed by Living Image software and presented as mean intensity.

Another biodistribution experiment was conducted as the same manner mentioned above. At the $36 \mathrm{~h}$ post-injection, the joint tissues were harvested. The positive frequency of the $\mathrm{Did}^{+} \mathrm{CD} 21^{+} / \mathrm{CD} 35^{+}$cells in the joints was measured by flow cytometry.

Therapeutic Efficacy In CIA: A $150 \mu \mathrm{L}$ of NEs@CitP/Rapa solution $(7.5$ mg/kg, CitP per body weight; $1 \mathrm{mg} / \mathrm{kg}$, Rapa per body weight) was injected intravenously on days $28,30,32,34,36,38,40$, and 42. Other groups, such as sterile PBS (control), NEs@OVA323-339 (3.5 mg/kg, OVA323-339 per body weight), NEs@CitP, and Free CitP/Rapa were treated on the same day. The healthy mice served as the naive control. Each group was comprised of 5 mice. Paw thickness and clinical score were evaluated 
every 3 days since the model establishment. On day 50, the mice were sacrificed, and the immunological and pharmacodynamic indicators were analyzed by the mentioned protocols.

The severity of arthritis was assessed by paw thickness and clinical score of the mice paws, ${ }^{3}$ mice were evaluated every 3 days since the model establishment, and images of mouse paws were taken at day 22. Specifically, the paw thickness and swelling of inflamed limbs were measured by a digital micro-caliper, and the thickness of mouse paws was measured from top to bottom of each inflamed paw. The clinical score ( 0 - 4) was given by a blinded researcher based on the following criteria: 0 , normal; 1, mild redness of ankle or tarsal joints; 2, mild redness and swelling extending from ankle to the tarsals; 3 , moderate redness and swelling from ankle to metatarsal joints; 4, severe redness and swelling encompassing the ankles, feet and digits.

Therapeutic Efficacy In AIA: 3 days after AIA induction, animals were randomly assigned to eight groups ( $n=6$ in each group). A $150 \mu \mathrm{L}$ of PBS (Control), NEs@CitP/Rapa solution $(7.5 \mathrm{mg} / \mathrm{kg}$, CitP per body weight; $1 \mathrm{mg} / \mathrm{kg}$, Rapa per body weight), NEs@CitP (7.5 mg/kg, CitP per body weight), NE@OVA323-339 (3.5 mg/kg, OVA323-339 per body weight), NEs@Rapa solution (1 mg/kg, Rapa per body weight), Free CitP/Rapa solution $(7.5 \mathrm{mg} / \mathrm{kg}$, CitP per body weight; $1 \mathrm{mg} / \mathrm{kg}$, Rapa per body weight) were administrated by tail vein once every 3 days for a total of 8 injections. Paw thickness and clinical score were evaluated every 3 days since the model establishment. On day 22, the mice sacrificed, and other immune and evaluation indexes of therapeutic effect were analyzed by the mentioned protocols. 
OVA-challenged experiments: CIA mice received treatment of NEs@CitP/Rapa as described above. Then the mice were immunized with ovalbumin (OVA) and CPG by subcutaneous injections into the back on day 50. Each injection contained a total of 10 $\mu \mathrm{g}$ OVA and $1 \mu \mathrm{g}$ CPG. The boost immunization was injected on the 7 th day of the first immunization. Blood samples were collected on 64th days from orbit and allowed clot at $4{ }^{\circ} \mathrm{C}$ for $2 \mathrm{~h}$, then serum was collected from the supernatant after centrifuging at 6,000 $\mathrm{g}$ for $10 \mathrm{~min}$. There are two other groups, including CIA mice received immunization of OVA and CPG but without treatment before, and CIA mice without treatment as the negative control. Each group comprised of 3 mice.

Antigen-specific antibody in serum: To detect the level of OVA-specific antibodies in serum, blood samples of CIA mice were collected on 64th days from orbit and allowed clot at $4{ }^{\circ} \mathrm{C}$ for $2 \mathrm{~h}$, then the serum was collected from the supernatant after centrifuging at $6,000 \mathrm{~g}$ for $10 \mathrm{~min}$. Serum levels of OVA-specific IgG, IgG1, and IgG2a antibodies were determined by ELISA. Polystyrene 96-well plates (Corning, USA) were coated with OVA protein $(10 \mu \mathrm{g} / \mathrm{mL}, 100 \mu \mathrm{L} /$ well $)$ diluted in coating buffer and incubated at $4{ }^{\circ} \mathrm{C}$ overnight. Mouse serum dilutions (1:3) were adopted, and the optical density (O.D.) was taken by a microtiter-plate spectrophotometer at $450 \mathrm{~nm}$.

Quantification of Serum and Synovium Cytokines: Mouse serum samples were collected at endpoint of therapeutic experiment (day 22 for AIA model, and day 50 for CIA model), and concentration of both pro-inflammatory cytokines (TNF- $\alpha$, IFN- $\gamma$, IL-1 $\beta$, IL-6) or anti-inflammatory cytokines (TGF- $\beta 1$, IL-10) were quantified by ELISA. Specifically, the whole blood was collected from mice orbit and allowed clot 
at $4{ }^{\circ} \mathrm{C}$ for $2 \mathrm{~h}$. Samples were centrifuged at $6,000 \mathrm{~g}$ for $10 \mathrm{~min}$ to collect serum from the supernatant. Serum samples were stored at $-20^{\circ} \mathrm{C}$, and then analyzed using various mouse ELISA kits.

Arthritic joints of CIA and AIA mice were harvested at endpoint of therapeutic experiment. The skin of the inflamed joints was removed, then the tissues were homogenized with an equal volume of RIPA Lysis Buffer (Beyotime, China) in the presence of a protease inhibitor PMSF (Beyotime, China) by homogenizer at $6000 \mathrm{rpm}$ with 30 seconds. Then the samples were centrifuged at 10,000 g for $30 \mathrm{~min}$ three times, and the supernatant was assayed for total protein level via a BCA assay. After adjusting the protein concentration of each sample to the same level $(\mathrm{OD} \pm 0.1)$, the concentration of cytokines was determined by ELISA Kits.

Histological Study: The collected joints were fixed in 4\% paraformaldehyde aqueous solution (v/v) for 2 days, then decalcified with PBS (PH 7.4) containing 15\% EDTA for 6 weeks. After decalcification, joint samples were transferred to $4 \%$ paraformaldehyde aqueous solution and prepared to histological analysis. Briefly, the sections were stained using hematoxylin and eosin (H\&E) to determine the level of cartilage erosion, and safranin O-fast green to evaluate the extent of cartilage destruction. For immunofluorescent analysis, samples were stained with primary antibodies against the indicated markers, including $\mathrm{T}$ cells $(\mathrm{CD} 3 \mathrm{e}$, clone 145-2C11, Invitrogen, USA) and APCs (CD40, Abcam, USA), to study the immunocyte in inflamed joints. All samples were stained with DAPI, and images were taken using a confocal microscope. 
Microcomputed Tomography: Paws from the experiment of therapeutic effect in CIA mice were removed on day 50, then micro-CT images were taken on an animal CT scanner (SCANCO Medical AG vivaCT 80, Switzerland). The instrument parameters were shown as below:

intensity $=145 \mu \mathrm{A}$, energy $=55 \mathrm{kV}$, voxel size $=15 \mu \mathrm{m}$, z-position slice $1=$ $56039 \mu \mathrm{m}, \mu$-scaling $=4096$.

Statistical Analysis: All data were analyzed by the software of GraphPad Prism 8. The results were shown as mean $\pm \mathrm{SD}$, unless noted otherwise. The significance of differences between two groups was analyzed by using Students' $t$-test, and the differences between multiple groups was were assessed by using one-way analysis of variance (ANOVA). The statistical difference was defined as $* P<0.05, * * P<0.01$, $* * * P<0.001, * * * * P<0.0001$.

\section{Supplementary Figures}

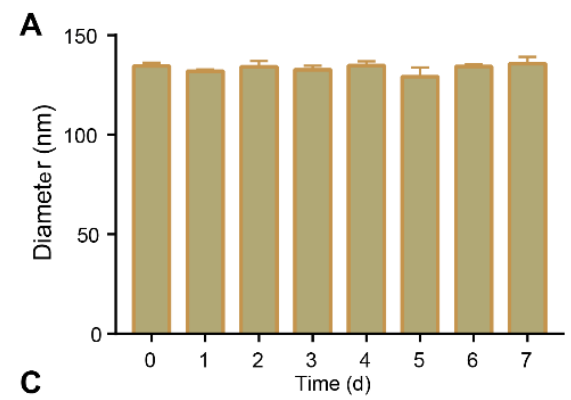

C

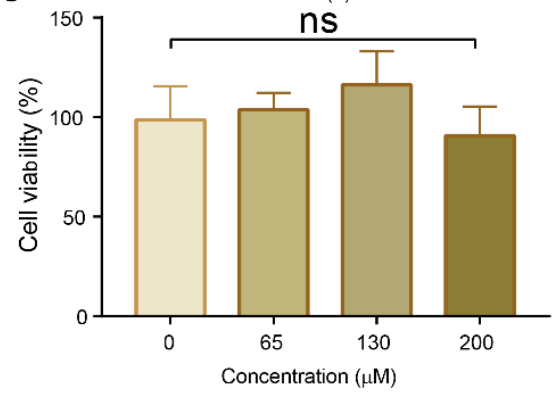

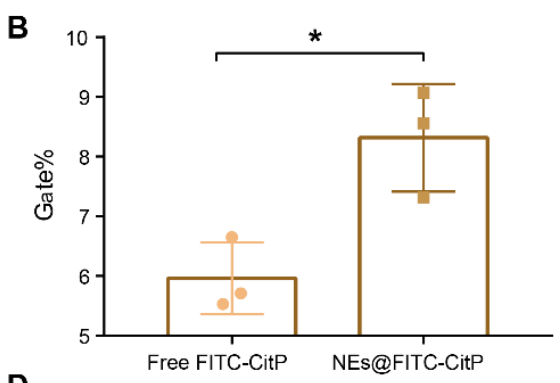

D

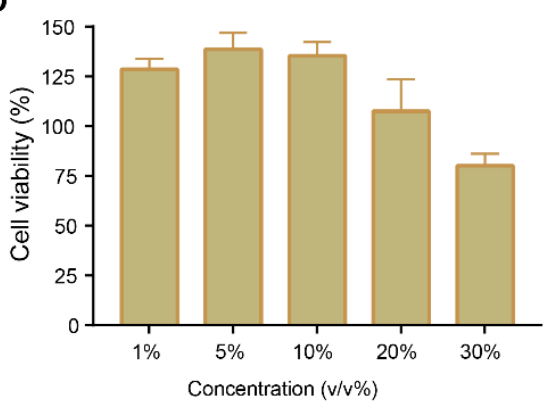

Figure S1. Characterization of NEs. (A) The stability of NEs@CitP/Rapa at $37{ }^{\circ} \mathrm{C}$. 
(B) The uptake efficiency of NEs@FITC-CitP on 3T3 cells. (C) Cytotoxicity of CitP peptide at different concentrations. DC2.4 cells were treated with CitP for $48 \mathrm{~h}$, and the cell viability was determined by CCK-8 assay. (D) RAW264.7 cells were treated with NEs containing various concentrations of ferric ion for $24 \mathrm{~h}$, and the cell viability was determined by CCK- 8 reagent. Data are presented as the mean $\pm \mathrm{SD}, \mathrm{n}=3 . * P<0.05$.

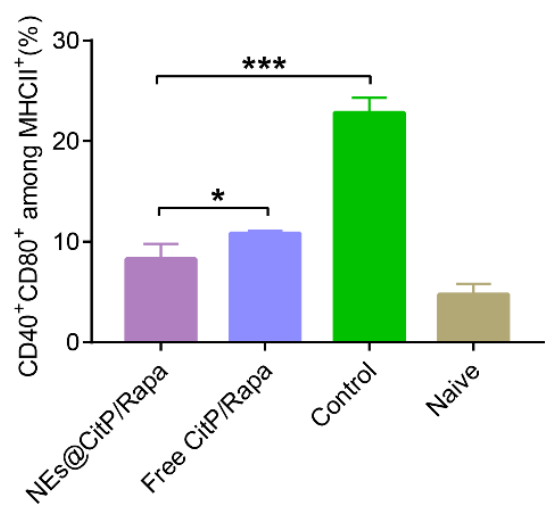

Figure S2. Tolerogenic APCs induced by NEs in vivo. The inhibition rate of APCs maturation for various groups. AIA model was established by immunization of Balb/c male mice. AIA mice received various formulations 8 times from 3 th to 17 th day with every other day. Healthy mice as Naive. AIA mice received intravenous injection of PBS (Control), NEs@CitP/Rapa, Free CitP/Rapa. rapamycin = 1 mg/kg (1.0 mg of rapamycin per body weight of mice), $\mathrm{CitP}=7.5 \mathrm{mg} / \mathrm{kg}$. AIA mice were sacrificed on day 20. The inflamed joints were harvested on 20th day, and single-cell suspensions were prepared by type IV collagenase. The inhibition rate of costimulatory molecules was analyzed using flow cytometry, and the frequency of $\mathrm{CD} 40^{+} \mathrm{CD} 80^{+}$cells was gated from $\mathrm{MHCII}^{+}$cells. Data are presented as the mean $\pm \mathrm{SD}, \mathrm{n}=3 .{ }^{*} P<0.05$, ***P $<$ 0.001 . 
A

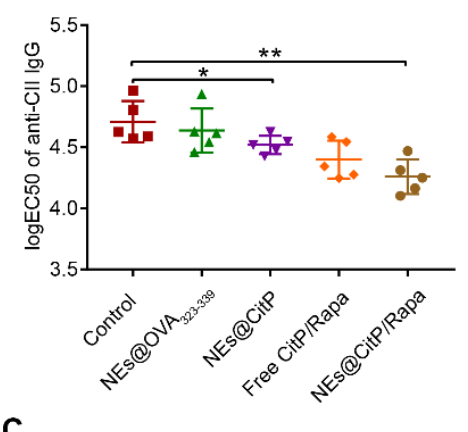

C

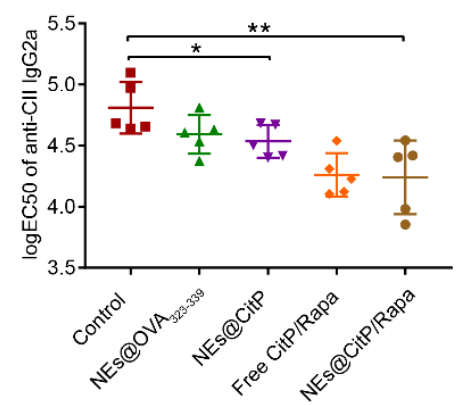

B

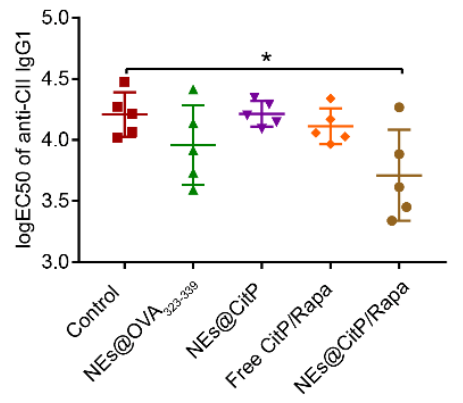

D

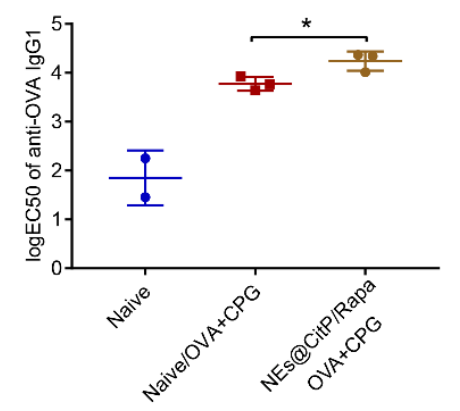

Figure S3. Therapeutic effect of various formulations in CIA mice. (A) The $\operatorname{logEC50}$ of anti-bCII (bovine type II collagen) IgG antibody in serum of various groups. The serum samples were collected on day 47 , and the anti-bCII specific antibodies were assayed by ELISA. (B) The $\operatorname{logEC} 50$ of anti-bCII IgG1 antibody in serum. (C) the $\operatorname{logEC50}$ of anti-bCII IgG2a antibody in serum. Data are showed as mean $\pm \mathrm{SD}, \mathrm{n}=5$. ${ }^{*} p<0.05,{ }^{* *} p<0.01$. (D) The $\log$ EC50 of OVA-specific IgG1 in serum of different groups after challenged by OVA antigen. Data are shown as mean $\pm \mathrm{SD}, \mathrm{n}=3 \cdot{ }^{*} p<$ 0.05 . 


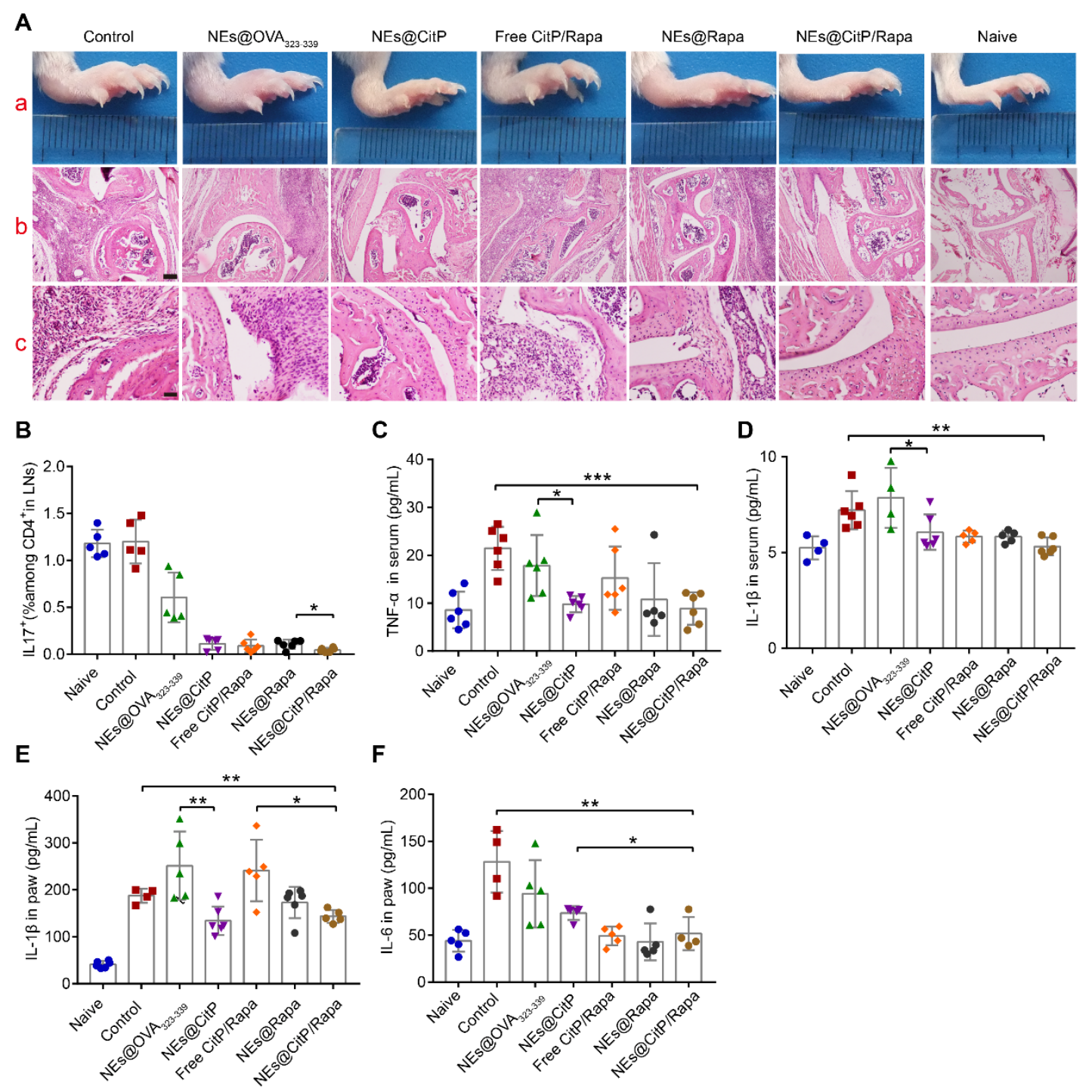

Figure S4. Therapeutic effect of various formulations in AIA mice. (A)

Representative photographs of paws for the different treatment groups on day 22 (a), and representative images of hematoxylin and eosin (H\&E) staining of ankle joint tissues extracted from mice of different treatment groups (b, c). b: scale bar $=200 \mu \mathrm{m}$. c: scale bar $=50 \mu \mathrm{m}$. (B) Frequency of Th17 cells in draining lymph nodes of hindpaws. Th17 cells were indicated as $\mathrm{IL}_{17} 7^{+}$cells among $\mathrm{CD}^{+}$gated from $\mathrm{CD}^{+}$. (C, D) Serum concentrations of pro-inflammatory cytokines TNF- $\alpha$ and IL-1 $\beta$, respectively. (E, F) Paw concentration of pro-inflammatory cytokines IL-1 $\beta$ and IL-6, respectively. 
The significance of the results was analyzed by using one-way ANOVA. Data are presented as the mean $\pm \mathrm{SD}, \mathrm{n}=4-6 .{ }^{*} P<0.05, * * P<0.01,{ }^{* * *} p<0.001$.

A

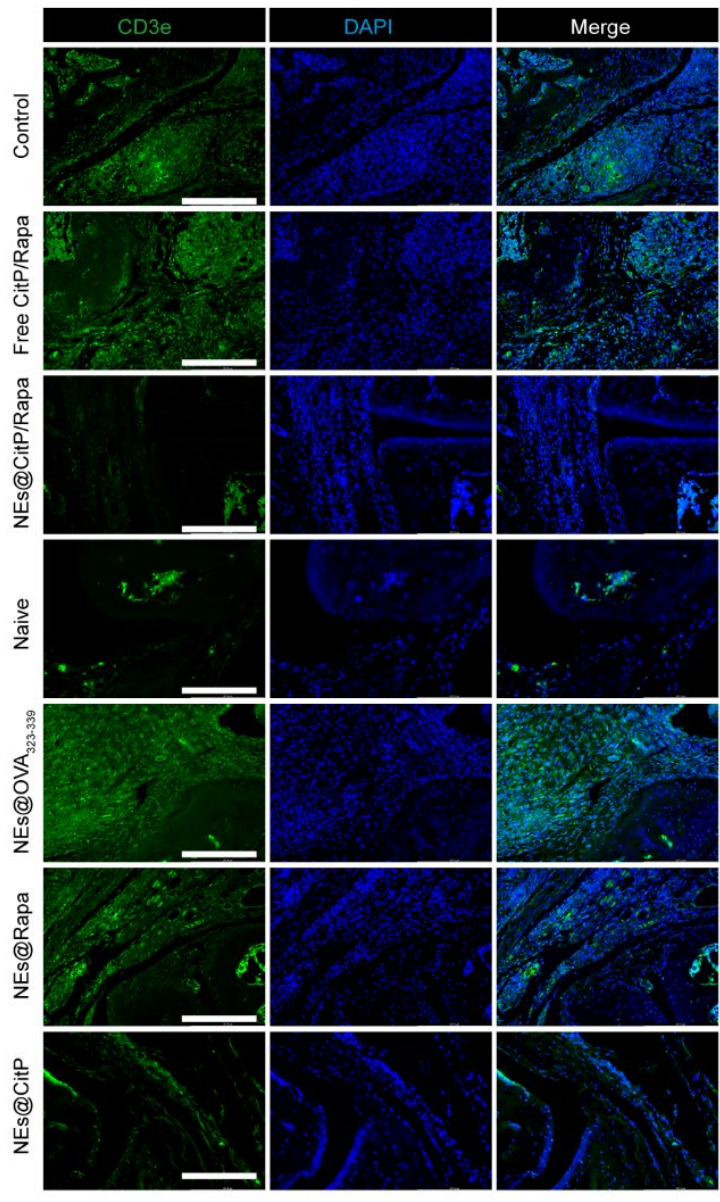

B

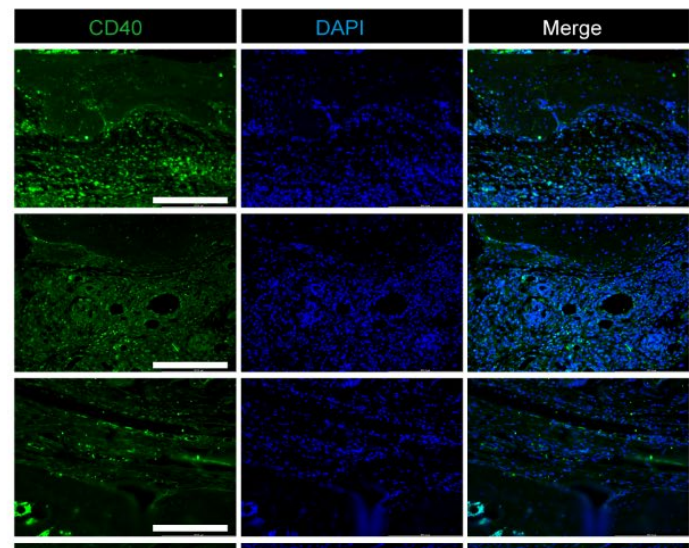

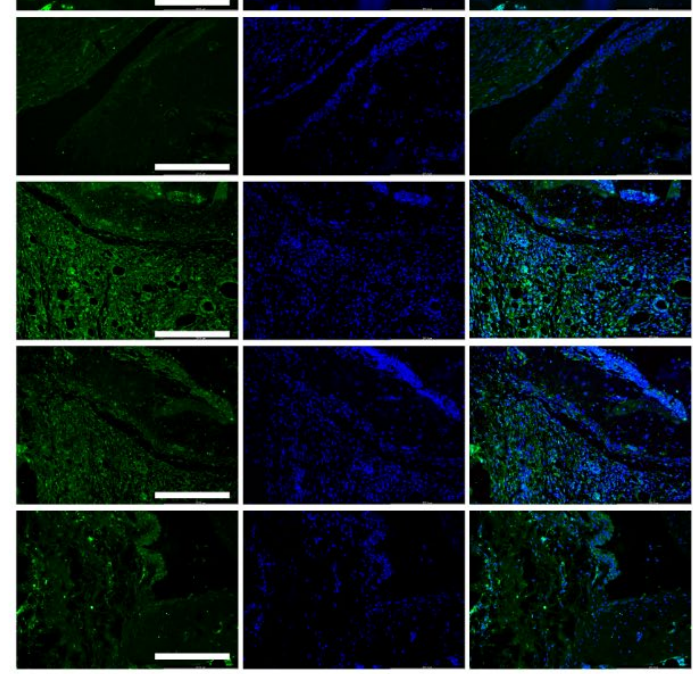

Figure S5. Immunofluorescent analysis of AIA mice after different treatments. The joint tissues from groups in Figure 5A showed the expression of CD3e/CD40 (green), DAPI (blue), and merge. A: CD3e. B: CD40. Scale bar $=200 \mu \mathrm{m}$. Paws were collected on day 22, and immunofluorescent analysis was conducted according to the protocol mentioned in methods.

\section{References}

(1) Lutz, M.; Kukutsch, N.; Ogilvie, A.; Rössner, S.; Koch, F.; Romani, N.; Schuler, G. Lutz MB, Kukutsch N, 
Ogilvie AL, Rossner S, Koch F, Romani N \& Schuler G. An advanced culture method for generating large quantities of highly pure dendritic cells from mouse bone marrow. J Immunol Methods223: 77-92. Journal of immunological methods 1999, 223, 77-92.

(2) Zhu, Y.; Xue, J.; Chen, W.; Bai, S.; Zheng, T.; He, C.; Guo, Z.; Jiang, M.; Du, G.; Sun, X. Albuminbiomineralized nanoparticles to synergize phototherapy and immunotherapy against melanoma. $J$ Control Release 2020, 322, 300-311.

(3) Zhang, Q.; Dehaini, D.; Zhang, Y.; Zhou, J.; Chen, X.; Zhang, L.; Fang, R. H.; Gao, W.; Zhang, L. Neutrophil membrane-coated nanoparticles inhibit synovial inflammation and alleviate joint damage in inflammatory arthritis. Nat Nanotechnol 2018, 13, (12), 1182-1190. 